\title{
Machine-Vision-Based and Inertial-Sensor-Supported Navigation System for the Minimal Invasive Surgery
}

\author{
Markus Haid ${ }^{1}$, Mustafa Kamil ${ }^{1}$, Thitipun Chobtrong ${ }^{1}$, Ersan Günes ${ }^{1}$ \\ ${ }^{1}$ Competence Center for Applied Sensor Systems (CCASS) \\ University of Applied Sciences Darmstadt, www.ccass.h-da.de
}

\begin{abstract}
:
The navigation of instruments, implants and patients in space has gained a broad acceptance for several years now. Especially in case of demand for minimal invasivity, navigated surgery has an enormous importance because of the decisive perceptual field expanding of the surgeons. The currently used systems in surgery are almost based on camera technology and image processing algorithms. The tracking solutions provide highly accurate three-dimensional position information of defined objects in space. On this basis, operation procedure can stepwise be assisted by a computer. Several clinical studies, by analyzing complaint rates of implanted joints and associated radiological images, could show that using navigation systems significantly decreases the implant failure.
\end{abstract}

Facing all improvements there are several disadvantages, which often are the reason for yet not navigated operating. High investment and maintenance costs, complexity in setup and operation and disturbances in the line of sight between camera and markers or leaving the permitted measurement field cause signal outages, loss of time, distraction and distrust of the technology.

The following study shows a technical analysis of current surgical navigation systems, discussing the existing problems and providing an improved procedure. Based on the state of the art and under consideration of given orthopedic guidelines, all requirements of a developing system are defined. Technically the work is based on the fusion of image processing routines with inertial sensors.

Key words: Inertial Navigation System, INS, Image Processing, Machine Vision, Minimal Invasive Surgery, Total Knee Replacement, TKR, Tracking, Navigation, Accelerometer, Gyroscope, Indoor, Low-cost, MEMS.

\section{Introduction}

The presented approach treats a specific application in the field of minimally invasive surgery (MIS). MIS especially targets for making as small incision during a surgical intervention as possible in order to reduce the rehabilitation time and the postoperative burden for the patient due to the injury.

In this sense technologically advanced computer-assisted navigation systems have gained access to the medical operating room. These systems are capable of providing valuable assistance information to the surgeon at accuracies down to the range of submillimeters. This information supports the surgical team where the human visual resolving abilities come to their limits. For example, navigation systems have their most advantage when the free visibility of anatomical details is not given, such as those are too tiny or the free line of site is interrupted. Navigation systems for computer-assisted surgery (CAS) mainly give information about the patient's posture on the operating table, the relations of surgical instruments to the patient's body and to each other as well as three-dimensional location and orientation information of relevant bones in space. Application examples are typically found in the fields of hip or knee arthroplasty. In both cases, it is of great importance for the postoperative result, that a significant spatial constellation of bones and joints exists after the operation. Particularly, these constellations are meant to fit the ideal orthopedic guidelines as those are known from literature.

Despite the extremely high precision of today's surgical navigation systems, there are various limitations which make it difficult to use them in everyday practice. Especially the complexity of operation is to be mentioned, as it causes delays and distraction to the medical staff and so leads to considerable loss of time.

The presented approach summarizes the implemented investigations on the feasibility of 
a novel system that is capable of keeping the advantages of today's navigation systems while compensating their limitations as described in the sections below.

\section{Navigating in the operating room}

Navigation systems in the operating room offer help to the surgeon in the selection of implants, suitable instruments, when removing damaged bone material and with aligning relevant bones and joints to each other allowing the desired postoperative results to be reached at highest rates of precision. Navigation without computerassisted alignment and positioning of implants and bones would inevitably result in a greater level of uncertainty, which is reflected in worse wear of the joint implant and postoperative discomfort.

The introduction of CAS went through a development curve beginning from the pure marketing function until today to everyday usability in operations and still developing for easier operation and lower maintenance costs. Currently available systems on the market offer a variety of advanced measurement and data processing functionalities for three-dimensional object tracking at accuracies up into the range of sub-millimeters. However, currently available surgical navigation systems suffer from several drawbacks which limit their usability or may even practically eliminate it.

Due to the low user-friendliness many systems bought by hospitals remain unused, so that operating is often carried out without navigation assistance in spite of a technical method is existing. The preoperative setup of the camera modules, their calibration and the registration of the boney structures into the system software is time-consuming and requires a specific order of work steps. That's why the medical personnel are required to find reliable and efficient techniques to overcome this issue, which creates a need for adequate training. In general it can be said, that the concentration of the primarily medically trained operator is diverted to technical issues. Ultimately, wrong configuration and erroneous systems are undesired sources of distraction and thus very critical during a surgical operation.

Further limitations are given with navigation systems that require CT-imaging before operation due to additional radiation exposure to the patients. The modern patient prefers CTfree methods as well as the organizational efforts for preparing an operation are more efficient when using imaging-free skeleton registration methods.
The consideration of using navigation systems doesn't begin in the operating room, but earlier when the investment decision is done by the clinics. This is where an additional disadvantage can be seen in today's CASsystems: they are expensive. Depending on number and type of required software modules the investment cost can get almost much higher than the pure system's price. Maintenance, training and cleaning are source of additional running costs.

In many cases, the investment risks associated with CAS are counter-financed by participation of an implant manufacturer to the investment. The risk here is that the patients' treatment isn't always implemented by means of the best suitable implant, but rather with the one that the manufacturer and system co-financer provides for the certain case. On the other hand, in this financing concept, a reduced system price could be of high interest for implant manufacturers.

\section{Concept}

Various studies in the field of total knee replacement (TKR) could show that axial misalignment of the leg after the insertion of an artificial knee joint is directly related to an increased rate of early failure. Here, the statistics show an acceptable tolerance range of the deviation of the mechanical leg axis of $\leq 3^{\circ}$. A study by Delp et al. reports further complications such as instabilities, postoperative movement of bones against each other, fractures or infections associated with wrong bone alignment. Therefore, and according to the relevant medical literature, it can be said, that a proper alignment of the lower extremity along the optimal mechanical axis of the leg is a very important parameter for increasing the lifetime of implants and that a way to tune this parameter during the surgical intervention is strongly required.

\section{Image processing-based determination of the mechanical leg axis}

Based on the front view of a leg skeleton an optimal axis for mechanical load can be defined by connecting the hip, the knee and the ankle joint with a straight line (cf. figure 1). Along this axis, the main load of the lower extremity is exerted by the body weight, and therefore a deviation of the knee joint center's position from the optimal axis directly causes uneven pressure distribution at the knee. Especially if this misalignment remains over a long period of time, damage of the joint can be expected. Deviations from the ideal axis are commonly 
known as "X-" or "O-leg" and are literately defined as "valgus" and "varus" constellations.

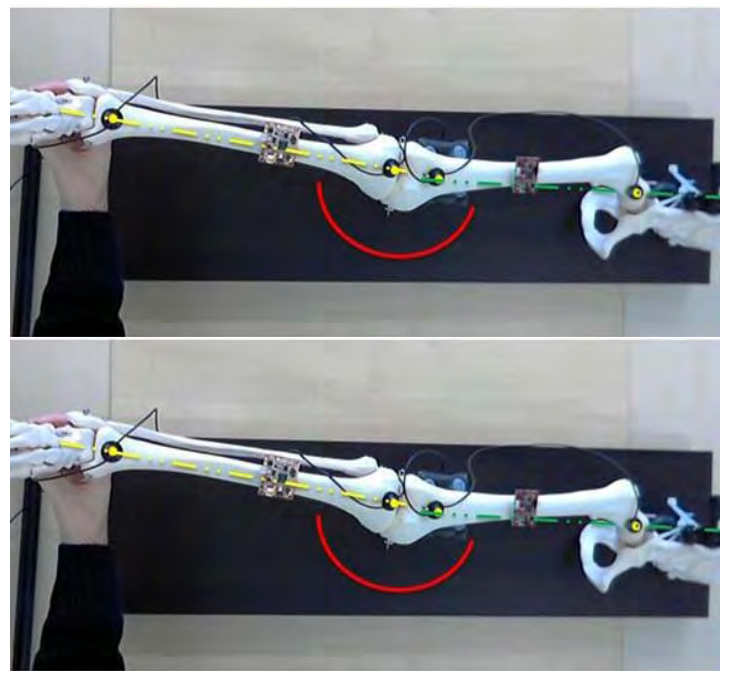

Fig. 1: Camera images from above showing a bad

(top) and an optimal alignment (bottom) of the leg's mechanical axis on a leg skeleton model

An assessment of the mechanical leg axis can automatically take place using an image processing algorithm. For this, the step of imaging acquisition is decisive as it has to meet certain quality criteria including optimal illumination, resolution and image distortion. The imaging can be made in terms of overall cost-reducing hardware solution using a standard miniature-size camera. Such modules meanwhile provide high quality images at very low hardware cost, so that also high-accuracy measurements can be obtained within certain bounds. Moreover, due to the use of a standard camera interface, this component is scalable, which allows higher quality cameras with better optics and more powerful electronics to be coupled to a proportional purchase price as an opportunity for further improvement of performance.

The system installation is designed to be simple and fast. First, a set of optical markers are to be fixed to designated points of the patient's leg. This is not trivial, since the bony structures of a human body are not necessarily predictable from outside without incision. Nevertheless, important bones and joint centers and can be located reliably by tactile sensing and rotating performed by the physician. These steps are already possible for a layman in approach, so they should be mastered by experienced staff without problems.

The optical markings can be fixed by temporary glue joints or loops with Velcro on the leg completely without injury. In the case of open access to the knee joint it is also possible to attach the markers directly to the implant. The attachment of the camera module follows at an optically suitable location. Here, an attachment straight above the surgical table or alternatively at a higher rank laterally is possible. Especially the second option is seen to be quite helpful, as the camera module can be attached to the same base (the operating table) as the measurement object (the patient's leg). For the first option it is also helpful to use the already existing lighting unit above the operation scene as a possible point of attachment. Alternatively, a lighting unit with an integrated camera could be conceivable. From the camera, a form of measurement data transfer to a processing unit is required, which can be done either by a direct cable connection or using a wireless interface.

The next step takes place within the image processing unit that should be able to provide calculation results in real-time. In the simplest case, a tiny computer unit can be combined with a screen display positioned on a movable table. The camera images must be searched for the positions of the optical markers and the line connecting the hip with the femoral knee center and the line connecting the tibial knee center and the ankle center can be defined (at least four markers are required). Subsequently, these lines are checked on their parallelism or, in other words, the angle between them is determined. This angle should be exactly 180 degrees by means of the definition of an ideal mechanical leg axis, so that at any deviation from this ideal value the user is informed with the necessary correction information including its direction. Alternatively the line between the hip and the ankle joint center can be determined in order to check, if the two points at the knee center are located on this line or not. If not, with the help of two angle measurements at the hip and the ankle the correction instructions can be calculated.

By using an image processing algorithm for checking the situation of the leg's axis, the described concept is susceptible to shading effects by objects in the line of sight between the camera and the markers. However, the concept can still be realized as the information about the leg's axis is only required at the beginning and the ending of the operation and the line of sight must only be free during these periods.

In the given application, only the joint markers are interesting in the image information, so that any other content is considered as uninteresting background information. Therefore, a suitable criterion has to be found in order to separate foreground and background pixels. A particularly strong contrast must be produced in 
captured scene in order to enable automated isolation. For this example this criterion is implemented by a contrasting color. The removal of the background is based on a set of color thresholds, containing three single channels (red, green and blue). The result of this segmentation is a binary image with a black background and small white circles at the marker's locations. The use of an edge detection algorithm then helps to determine their coordinates in the image, so that every marker can be assigned to a specific pixel and then to a specific label. The obtained coordinates are then used for connecting the two vectors as described above, so that the determination of the desired angle is possible. The latter is finally compared to the orthopedic ideal and so can be assigned to categories of "good", "bad" or any other sub-categories (cf. figure 2, 3).

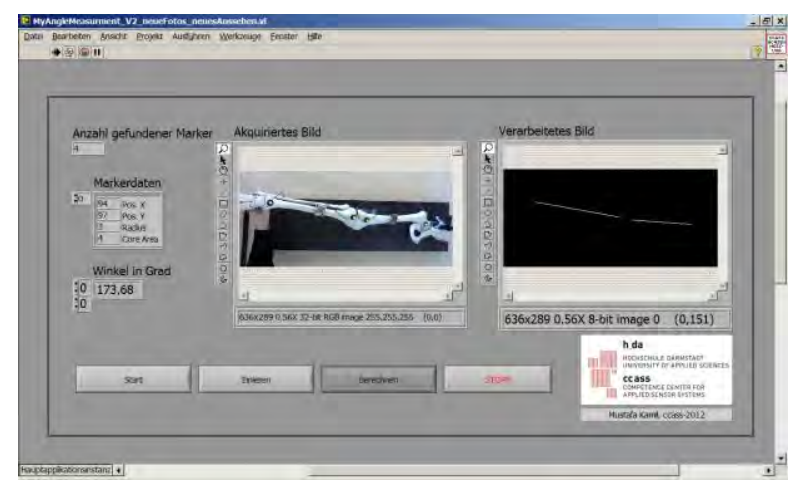

Fig. 2: Results of image processing showing the mechanical axis, marker coordinates, marker count, and calculated angle between femur and tibia bones

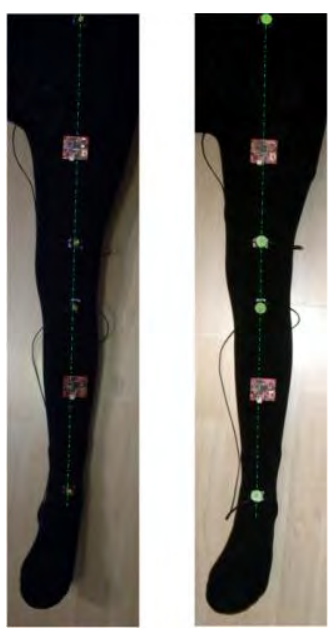

Fig. 3: Machine-vision-based determination of the mechanical axis and inertial inspection of the knee's range of motion, demonstration on a human leg

\section{Inertial measurement of knee motion range}

The evaluation of the knee's motion range after the insertion of the polyethylene implant in-lay can be implemented with an angle measurement at femur and tibia bones around the center the knee. Such a test can help the surgeon in selecting the optimal in-lay already during the operation and so can minimize the probability of postoperative complaints.

Guideline values for the ideal range of motion according to recognized literature lay between 120 and 150 degrees between maximum leg extension and maximum flexion. This range can be determined with the help of low-cost inertial acceleration sensors.

The term "inertial" derives from the Latin "Inertia". What is meant here is the inertia of a tiny mass, the so-called "inertial mass" inside the sensor, which shows a delayed displacement to the sensor housing in case of its acceleration. Based on this operating principle miniature-size and low-cost sensor systems exist for measuring acceleration and angular rates at moving bodies. In larger designs, such sensors can be found e.g. in navigation of aircraft or ships as well as in small variations in crash detection systems in automotive applications. Low-cost inertial navigation systems are almost based on micromachined sensors (also: MEMS for "microelectro-mechanical system") and are built with an orthogonal constellation of both three accelerometers and three angular rate sensors, creating a Cartesian coordinate system with six degrees of freedom.

In order to allow long-term application accuracy in spite of the sensor drift that low-cost inertial sensors have, a numerical integration must be avoided. This is feasible, especially for the implementation of an inclination measurement system, since accelerometers also react on the static gravitational acceleration with a deflection of the inner mass. The extent of deflection is directly dependent from the angle between the inertial measurement platform and the gravity vector, similar to the principle of a bubble level.

Based on this principle, two rotational degrees of freedom can be used, so a two-dimensional inclination measurement is obtained. For the third angle (yaw) the principle isn't usable due to its independence from gravity, which in turn is the reason for using the camera measurement.

Inertial inclination measurement is nowadays massively applied in various electronic devices for intuitive MMl-concepts game consoles, smart phones, tablet PCs etc. Two first massproduced devices with this technology are the 
Nintendo Wii console and Apple's iPhone. One of the secrets of this technology's success lies primarily in the possibility of integration-free angle calculation which therefore is stable over long periods of time. Also, the angle resolution can be obtained at highest accuracy rates, lying far under the limit of a sub-degree.

The angle calculation can be achieved the easiest with the accelerometer's signal and a arcsine function. Although this is mathematically correct, this method isn't adopted here because the measurement would have an angle-dependent sensitivity due to the deviation function of the arcsine. A constant sensitivity over the entire measurement range is reached using the arctangent function when and the signal triad of a three-dimensional accelerometer.

The implementation of the inclination measurement is validated with comparison to a high-accuracy optical navigation system starting at a slight negative slope of about 20 degrees and increasing in five positively directed steps to about 140 degrees, according to the literately defined normal motion range of the knee joint. Highest importance is given to the angle measurements at the beginning and at the end of the measurement range, as those define the joint motion. The hardware configuration and the results of this experiment are shown in figure 4 and figure 5 .

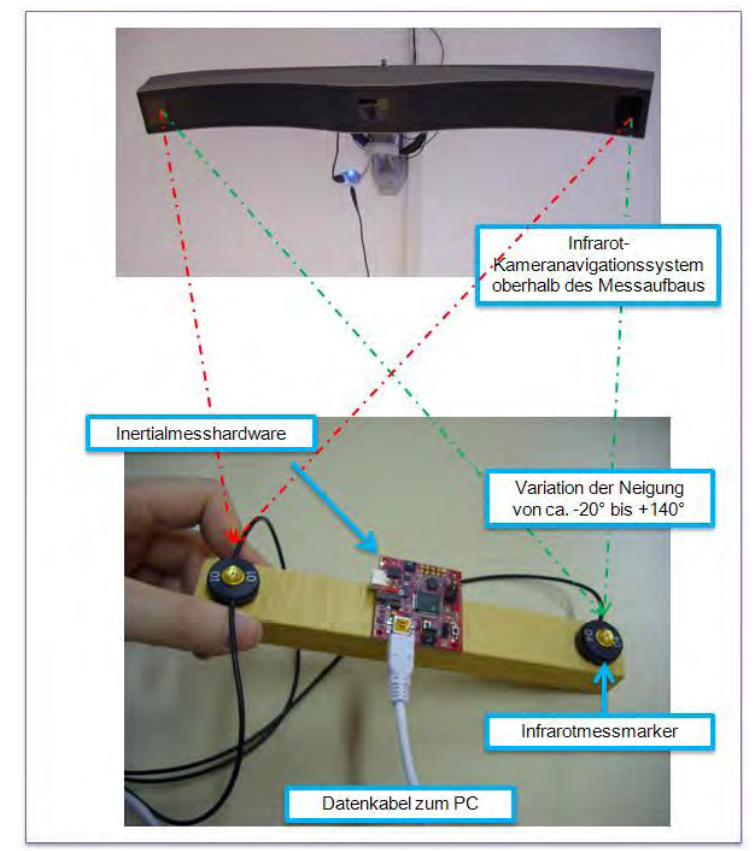

Fig. 4: Experimental assembly for the validation of the inertial knee motion measurement

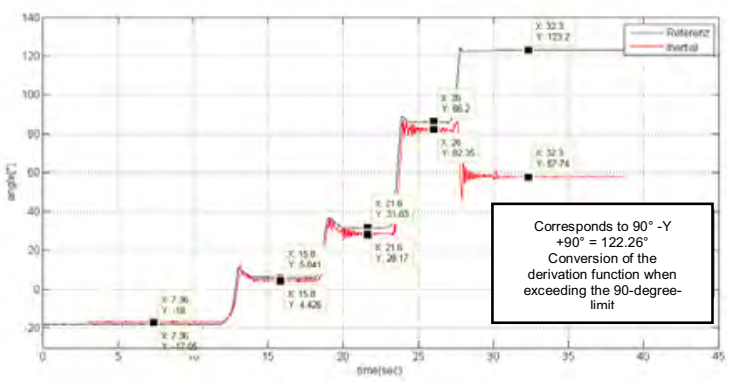

Fig. 5: Results of the inertial range of motion measurement in comparison to high-end infra-red camera reference system

In figure 5 a step-like plot of the calculated bone inclination can be seen, which was expected from the experimental procedure as described above. The last measurement step shows a specialty in gravity based angle calculation. Due to the sine-based behavior of the measured acceleration value with respect to the angle of inclination, a change of sign is observed after exceeding the 90 degrees mark as a result of the derived sine function (the cosine function has a zero point at $+90^{\circ}$ ). Therefore, all angle values above this limit must be interpreted accordingly, ideally by taking the signal's derivative function into account.

As shown in figure 5 , is the largest deviation of the inertial angle measurement in comparison to the optical reference system is 3.85 degrees. The smallest deviation is 0.94 degrees. The average deviation is 2.163 degrees. The limits of the measuring range have the most relevance and show the smallest differences against the reference measurement of each less than one degree.

\section{Conclusion}

In our study, novel methods were presented for application in CAS-systems. The resulting fine concept was validated successfully and can be the basis for the realization of a first prototype. Overall, this first study could be completed satisfactorily and the feasibility question for new simple and more cost-efficient measurement principles for CAS was answered positively.

The next step would be to gain additional experience in the specific application field, more in-depth feasibility studies and further evaluations of additional sensors that potentially can enhance the presented concepts. Further questions are given in what precision the markers can be attached on the joint centers and whether there are ways to support this process' reproducibility by additional measurements. A prototype implementation and further 
testing in an authentic operating environment is also desirable.

\section{Future Vision: Full inertial navigation system for shading-free determination of the mechanical axis}

It is possible to extend the inertial approach with a system that can also offer entire resistance to interruptions of line of sight. This can be achieved with the integration of acceleration, yaw rate and electronic compass sensors into one common algorithm. An appropriate signal processing translates all sensor information into a stable and accurate three-dimensional position and orientation measurement. The use of an INS can thus be advanced so that hereby a redundancy is achieved in the case of a broken line of sight between the camera and markers. This approach is already in development at the CCASS and will be prospectively presented in coming up paper publications.

\section{References}

[1] Wolfgang Rüdiger Hepp, Hans U. Debrunner: Orthopädisches Diagnostikum (2004).

[2] R. Haaker, W. Konermann: Navigation und Robotik in der Gelenk- und Wirbelsäulenchirurgie (2003).

[3] Jens Agneskirchner, Philipp Lobenhoffer: Kniegelenknahe Osteotomien (2007).

[4] Lutz-Kovacs-Electronics: LUKOtronic Motion Analysis System AS 200, System Description (2006).

[5] Markus Haid: Verbesserung der referenzlosen inertialen Objektverfolgung zur Low-cost IndoorNavigation durch Anwendung der KalmanFilterung, Dissertation, Universität Siegen (2004).

[6] Aesculap AG: Aesculap® Patienteninformation, Knieoperation mit dem OrthoPilot $\AA$ Navigationssystem (2010), http://www.aesculapextra.net/public/frame_doc_index.html?med_id=1 00051825 (2011).

[7] Southern Orthopaedics: Computer-Assisted Surgery (CAS) with Minimally-Invasive Surgery (MIS) Total Knee Replacement using VectorVision® knee TKR Navigation (2005), http://www.so.com.au/Patient_information/conditi ons/Knee/Tkr/tkr\%20pages/knee_cas.htm (2012). 